\title{
Change in lipid components in the adipose and liver tissues of regucalcin transgenic rats with increasing age: Suppression of leptin and adiponectin gene expression
}

\author{
MASAYOSHI YAMAGUCHI and TAEKO NAKAGAWA \\ Laboratory of Endocrinology and Molecular Metabolism, Graduate School of Nutritional Sciences, \\ University of Shizuoka, 52-1 Yada, Suruga-ku, Shizuoka 422-8526, Japan
}

Received March 12, 2007; Accepted April 23, 2007

\begin{abstract}
Regucalcin plays a multifunctional role as a regulatory protein in intracellular signaling pathway in many cell types. Regucalcin transgenic (TG) rats have been shown to experience hyperlipidemia with increasing age. This study was undertaken to determine whether lipid components in the adipose and liver tissues are changed in regucalcin TG rats in vivo. Female regucalcin TG rats were used at 7 or 50 weeks of age. Serum triglyceride or HDL-cholesterol concentrations were significantly increased in 7-week-old regucalcin TG rats as compared with those in 7-week-old normal rats. Serum triglyceride, total cholesterol, HDL-cholesterol, or free fatty acid concentrations were significantly increased in 50-weekold regucalcin TG rats. Meanwhile, triglyceride content in the adipose tissues was significantly increased in 50-week-old regucalcin TG rats, while the free fatty acid content was not significantly changed. Triglyceride, total cholesterol, or free fatty acid content in the liver tissues was significantly decreased in 50-week-old regucalcin TG rats. Liver glycogen content was significantly decreased in 7- or 50-week-old regucalcin TG rats. In addition, regucalcin mRNA and its protein levels were seen in the adipose tissues of normal rats. Those levels were not significantly changed in regucalcin TG rats at 50 weeks of age. Leptin mRNA expression in the adipose or liver tissues was significantly decreased in 50-week-old regucalcin TG rats. Adiponectin mRNA levels were not significantly changed in the adipose tissues of 50-week-old regucalcin TG rats, while the levels were significantly decreased in the liver tissues. This study demonstrates that the disorder of lipid metabolism in the adipose and liver tissues is induced in regucalcin TG rats with aging, and that the gene expression of leptin or adiponectin is suppressed in TG rats.
\end{abstract}

Correspondence to: Dr Masayoshi Yamaguchi, Laboratory of Endocrinology and Molecular Metabolism, Graduate School of Nutritional Sciences, University of Shizuoka, 52-1 Yada, Suruga-ku, Shizuoka 422-8526, Japan

E-mail: yamaguch@u-shizuoka-ken.ac.jp

Key words: regucalcin, hyperlipidemia, lipid metabolism, leptin, adiponectin

\section{Introduction}

Regucalcin was discovered in 1978 as a calcium-binding protein that does not contain the EF-hand motif of the $\mathrm{Ca}^{2+}$ binding domain (1-5). The name regucalcin was proposed for this $\mathrm{Ca}^{2+}$-binding protein, which regulates the $\mathrm{Ca}^{2+}$ effect on liver cell function $(4,6)$. In recent years, regucalcin has been demonstrated to play a multifunctional role as a regulatory protein in intracellular signaling processes in many cell types (reviewed in refs. 6-9).

The gene of regucalcin is highly conserved in vertebrate species (10). The rat and human regucalcin genes are localized on chromosome $X(11,12)$. Regucalcin mRNA and its protein are greatly present in liver and kidney cortex $(13,14)$. AP1, NF1-A1, and RGPR-p117, a novel protein, have been found to be transcriptional factors for the enhancement of regucalcin gene promoter activity (15-18). The expression of regucalcin mRNA is mediated through a $\mathrm{Ca}^{2+}$-dependent signaling mechanism $(16,19,20)$.

Regucalcin has been demonstrated to play a role in the maintenance of intracellular $\mathrm{Ca}^{2+}$ homeostasis, the inhibitory regulation of various $\mathrm{Ca}^{2+}$-dependent protein kinases, tyrosine kinases, protein phosphatases, and nitric oxide synthase (6-9). Regucalcin has a suppressive effect on DNA and RNA syntheses in the nucleus of proliferative cells $(21,22)$. Overexpression of regucalcin modulates tumor-related gene expression and suppresses cell proliferation in cloned rat hepatoma H4-II-E cells $(23,24)$. Moreover, overexpression of regucalcin has been shown to have a suppressive effect on cell death and apoptosis induced with stimulation of various factors in cloned rat hepatoma H4-II-E cells (25-27). Regucalcin has been proposed to play a physiologic role in the maintenance of homeostasis of cellular response for cell stimulation (reviewed in ref. 12).

Regucalcin transgenic (TG) rats were generated to determine the regulatory role of endogenous regucalcin in vivo (25). Bone loss has been demonstrated in regucalcin TG rats, indicating that regucalcin plays a role in the regulation of bone metabolism (29). Moreover, hyperlipedemia has been shown to occur in regucalcin TG rats with increasing age (30).

This study was undertaken to determine whether lipid components are changed in the adipose and liver tissues of regucalcin TG rats. 


\section{Materials and methods}

Chemicals. Chemicals were of reagent grade and were obtained from Sigma Chemical (St. Louis, MO, USA), Wako Pure Chemical Industries (Osaka, Japan) and Sigma (St. Louis).

Regucalcin transgenic (TG) rats. Regucalcin TG rats (SpragueDawley) were generated previously (28). The TG rats (5 weeks old) were obtained from Japan SLC Inc. (Hamamatsu, Japan). To determine transgenic copy number, genomic DNA was isolated from tails of TG rats and amplified by reverse transcription-polymerase chain reaction (RT-PCR) using primer sets that recognized two different regions of the regucalcin cDNA (10). Primers huRC-1 (5'-GGAGGCTATG TTGCCACCATTGGA-3') and huRC-2 (5'-CCCTCCAAA GCAGCATGAAGTTG-3') amplified a fragment containing the regucalcin cDNA that was present in the transgene sequence, but absent in the wild-type (wt). Regucalcin TG rats were fed commercial laboratory chow (solid) containing $57.5 \%$ carbohydrate, $1.1 \%$ calcium, and $1.1 \%$ phosphorus at room temperature $\left(25^{\circ} \mathrm{C}\right)$ and were allowed distilled water freely.

Biochemical determination. Animals were bled by cardiac puncture under light ether anesthesia at 7 or 50 weeks of age. Blood samples were centrifuged $30 \mathrm{~min}$ after collection, and the serum was separated. Serum was stored at $-80^{\circ} \mathrm{C}$ until assay. The adipose or liver tissues were removed and washed with ice-cold $0.25 \mathrm{M}$ sucrose solution and immediately frozen at $-80^{\circ} \mathrm{C}$. Serum glucose, free fatty acid, triglyceride, HDLcholesterol, or total cholesterol concentrations were determined using an assay kit (Wako Pure Chemical Co.).

To determine lipid components in the adipose or liver tissues, these tissues were cut into small pieces, suspended 1:9 (weight: volume) in $0.25 \mathrm{M}$ sucrose solution, and homogenized in a Patter-Elvehjem homogenizer with a Teflon pestle. The homogenate was spun at 5,500 $\mathrm{x} \mathrm{g}$ in a refrigerated centrifuge for $10 \mathrm{~min}$ to remove the mitochondria. The $5,500 \mathrm{x} \mathrm{g}$ supernatant fraction was pooled to assay lipid components and regucalcin levels. Lipid components in the 5,500 x g supernatant fraction obtained from the homogenization of adipose or liver tissues were determined using the assay kit (Wako Pure Chemical Co.). Lipid components were expressed as $\mathrm{mg}$ of lipid components/g of wet tissues.

Liver glycogen was determined using the method of Good et al (31). Glycogen content was expressed as mg of glucose/ $\mathrm{g}$ of wet liver tissues.

Western blot analysis. Aliquots of adipose homogenate supernatant (30 $\mu \mathrm{g}$ of protein) from normal rats or regucalcin transgenic rats were mixed with $5 \mathrm{X}$ Laemmli sample buffer, boiled for $5 \mathrm{~min}$, and resolved by $12 \%$ SDS-PAGE (32). The proteins were then transferred onto a polyvinylidene difluoride

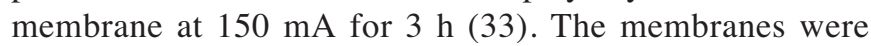
incubated with a polyclonal rabbit anti-regucalcin antibody (11), which was diluted 1:2,000 in $10 \mathrm{mM}$ Tris-HCl, $\mathrm{pH} 8.0$, containing $150 \mathrm{mM} \mathrm{NaCl}, 0.1 \%$ (wt./vol.) Tween-20 (washing buffer), and $5 \%$ (wt./vol.) skim milk for $1 \mathrm{~h}$. The membranes were incubated and washed four times with washing buffer. Then, the membranes were incubated for $1 \mathrm{~h}$ with horseradish peroxidase-linked anti-rabbit $\mathrm{IgG}$, which was diluted 1:5,000 in washing buffer containing 5\% (wt./vol.) skim milk, and again they were washed. Detection of the protein bands was performed using the enhanced chemiluminescent kit following the manufacturer's instructions. The exposure time of the membranes onto film was $10 \mathrm{~min}$. The molecular size of the detecting protein was determined by running the standard protein molecules of known sizes in parallel. Protein concentration in the adipose and liver tissues was determined using the method of Lowry et al (34).

Determination of specific mRNA by RT-PCR. Total RNAs were prepared as described previously (35). Aliquots of adipose or liver tissues were homogenized in a buffer solution containing $4 \mathrm{M}$ guanidinium thiocyanate, $24 \mathrm{mM}$ sodium citrate ( $\mathrm{pH} 7.0$ ), $0.5 \%$ sarcosyl, and isoamyl alcohol, and the phases were separated by centrifugation at $10,000 \mathrm{x} \mathrm{g}$ for $20 \mathrm{~min}$ at $4^{\circ} \mathrm{C}$. RNA located in the aqueous phase was precipitated and pelleted by centrifugation, and the pellets were redissolved in diethylpyrocarbonate-treated water.

RT-PCR was performed with a Titan ${ }^{\mathrm{TM}}$ one tube RT-PCR kit (Roche Molecular Biochemicals) as recommended by the supplier to investigate the gene expression of regucalcin, leptin, adiponectin, or glyceraldehyde-3-phosphate dehydrogenase (G3PDH). The generated primers were based on the published rat sequences. Primers for regucalcin cDNA were 5'-AGATG AACAAATCCCAGAT-3' (sense strand, positions 618-696) of cDNA sequence (10), and 5'- TCACCCTGCATAGGAAT AT-3' (anti-sense strand, positions 906-924). The pair of oligonucleotide primers was designed to amplify a 307-bp sequence from the mRNA of rat regucalcin cDNA.

Primers for leptin cDNA were 5'-GGCTTTGGTCCT ATCTGTCC-3' (sense strand, positions 91-111) of cDNA sequence (36), and 5'-ATAGACTGCCAGGGTCTGGT-3' (anti-sense strand, positions 306-325). The pair of oligonucleotide primers was designed to amplify a 224-bp sequence from the mRNA of rat leptic cDNA.

Primers for adiponectin cDNA were 5'- GAGAAGGGA GAGAAGGGAGA-3' (sense strand, positions 211-230) of cDNA sequence (37), and 5'-GTAGTAGAGCCCCGGAA TGT-3' (anti-sense strand, positions 498-517). The pair of oligonucleotide primers was designed to amplify a 307-bp sequence from the mRNA of rat adiponectin cDNA.

Primers for G3PDH cDNA were 5'- CATGTAGGCCAT GAGGTCCACCAC-3' (sense strand) 5'- CATGTAGGCCA TGAGGTCCACCAC-3' (anti-sense strand) from the G3PDH amplimer set (Clontech, Palo Alto, CA).

RT-PCR was performed using reaction mixture $(20 \mu \mathrm{l})$ containing $1 \mu \mathrm{g}$ for leptin and adiponectin mRNAs or $2 \mu \mathrm{g}$ for regucalcin of total RNAs, supplied RT-PCR buffer, Titam enzyme mix (AMV and Expand ${ }^{\mathrm{TM}}$ High Fidelity), $0.2 \mathrm{mM}$ deoxynucleotide triphosphate, $5 \mathrm{mM}$ dithiothreitol, $5 \mathrm{U}$ RNase inhibitor, 2.5 U TaqDNA polymerase, and $0.3 \mu 1$ primers. Samples were incubated at $50^{\circ} \mathrm{C}$ for $30 \mathrm{~min}$ and at $94^{\circ} \mathrm{C}$ for $2 \mathrm{~min}$, and then amplified for 35 cycles under the following conditions: denaturation for $30 \mathrm{sec}$ at $94^{\circ} \mathrm{C}$, annealing for $30 \mathrm{sec}$ at $58^{\circ} \mathrm{C}$ or $60^{\circ} \mathrm{C}$ and extension for $60 \mathrm{sec}$ at $68^{\circ} \mathrm{C}$. The amplified PCR products were separated by electrophoresis on a $1.5 \%$ agarose gel, visualized by ethidium bromide staining and quantitated using a densitometer. 
Table I. Change in serum findings in regucalcin transgenic (TG) rats.

\begin{tabular}{lcc}
\hline Serum findings & Normal & Regucalcin TG \\
\hline 7-week-old rats & & \\
Triglyceride $(\mathrm{mg} / \mathrm{dl})$ & $56.41 \pm 5.52$ & $86.66 \pm 7.41^{\mathrm{a}}$ \\
Total cholesterol $(\mathrm{mg} / \mathrm{dl})$ & $75.80 \pm 3.10$ & $91.94 \pm 5.93$ \\
HDL-cholesterol $(\mathrm{mg} / \mathrm{dl})$ & $142.87 \pm 6.50$ & $186.62 \pm 1.97^{\mathrm{b}}$ \\
Free fatty acid $(\mu \mathrm{Eq} / \mathrm{l})$ & $0.30 \pm 0.01$ & $0.31 \pm 0.03$ \\
Glucose $(\mathrm{mg} / \mathrm{dl})$ & $137.34 \pm 10.04$ & $129.95 \pm 4.58$ \\
& & \\
50-week-old rats & & \\
Triglyceride $(\mathrm{mg} / \mathrm{dl})$ & $170.09 \pm 9.10$ & $238.57 \pm 16.90^{\mathrm{c}}$ \\
Total cholesterol (mg/dl) & $81.36 \pm 4.22$ & $115.68 \pm 5.86^{\mathrm{b}}$ \\
HDL-cholesterol $(\mathrm{mg} / \mathrm{dl})$ & $183.70 \pm 5.99$ & $250.88 \pm 16.36^{\mathrm{c}}$ \\
Free fatty acid $(\mu \mathrm{Eq} / \mathrm{l})$ & $0.42 \pm 0.03$ & $0.61 \pm 0.06^{\mathrm{c}}$ \\
Glucose $(\mathrm{mg} / \mathrm{dl})$ & $156.41 \pm 0.34$ & $161.70 \pm 5.00$ \\
\hline
\end{tabular}

Normal (wild-type) or regucalcin TG rats were sacrificed by bleeding at 7 or 50 weeks of age. Each value is the mean \pm SEM of six rats. ${ }^{\mathrm{a}} \mathrm{p}<0.01$ compared with the value obtained from normal rats. ${ }^{b} \mathrm{p}<0.025$ compared with the value obtained from normal rats. ${ }^{c} \mathrm{p}<0.05$ compared with the value obtained from normal rats.

Statistical analysis. Data are expressed as the mean \pm standard error of the mean (SEM). Statistical differences were analyzed using Student's t-test. A p-value $<0.05$ was considered to indicate statistically significant difference. Also, we used an ANOVA multiple comparison test to compare the treatment groups.

\section{Results}

Change in lipid components in adipose or liver tissues in regucalcin transgenic (TG) rats. The change in serum lipid concentrations in regucalcin TG rats is shown in Table I. Normal (wild-type) or regucalcin TG rats were sacrificed by bleeding at 7 or 50 weeks of age. Serum triglyceride or HDLcholesteral concentrations were significantly increased in 7-week-old regucalcin TG rats as compared with those in 7-week-old normal rats. Serum triglyceride, total cholesterol, HDL-cholesterol, or free fatty acid concentrations were significantly increased in 50-week-old regucalcin TG rats as compared with those in 50-week-old normal rats. Serum glucose concentrations were not significantly changed in 7or 50-week-old regucalcin TG rats.

Triglyceride content in the adipose tissues was significantly increased in 50-week-old regucalcin TG rats as compared with that in 50-week-old normal rats (Fig. 1). Free fatty acid content in the adipose tissues was not significantly changed in 7- or 50-week-old regucalcin TG rats (Fig. 2).

Triglyceride (Fig. 3), total cholesterol (Fig. 4), or free fatty acid (Fig. 5) content in the liver tissues was significantly decreased in 50-week-old regucalcin TG rats as compared with those in 50-week-old normal rats. Such a decrease was not seen in 7-week-old regucalcin TG rats (Figs. 3-5). Liver

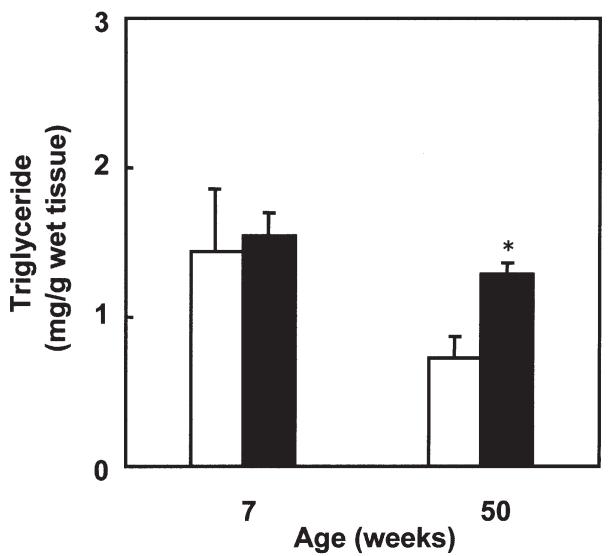

Figure 1. Change in triglyceride content in the adipose tissues of normal or regucalcin transgenic (TG) rats. Rats were sacrificed by bleeding at 7 or 50 weeks of age. Each value is the mean \pm SEM of six rats. ${ }^{*} p<0.01$ compared with the control value from normal rats. White bars, normal rats; black bars, TG rats.

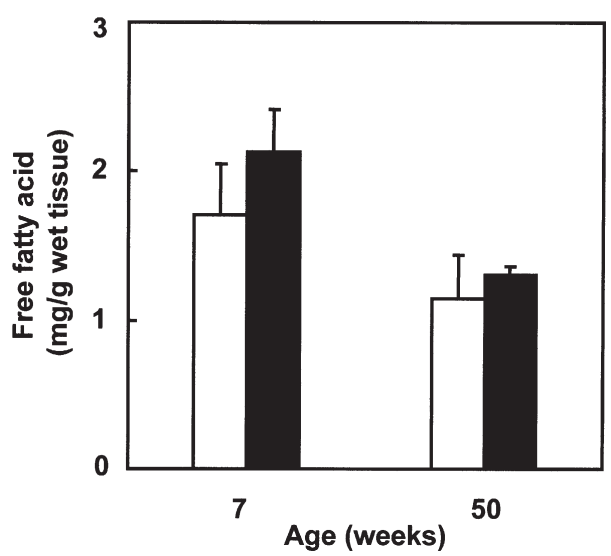

Figure 2. Change in free fatty acid content in the adipose tissues of normal or regucalcin transgenic (TG) rats. Rats were sacrificed by bleeding at 7 or 50 weeks of age. Each value is the mean \pm SEM of six rats. Data were not significant. White bars, normal rats; black bars, TG rats.

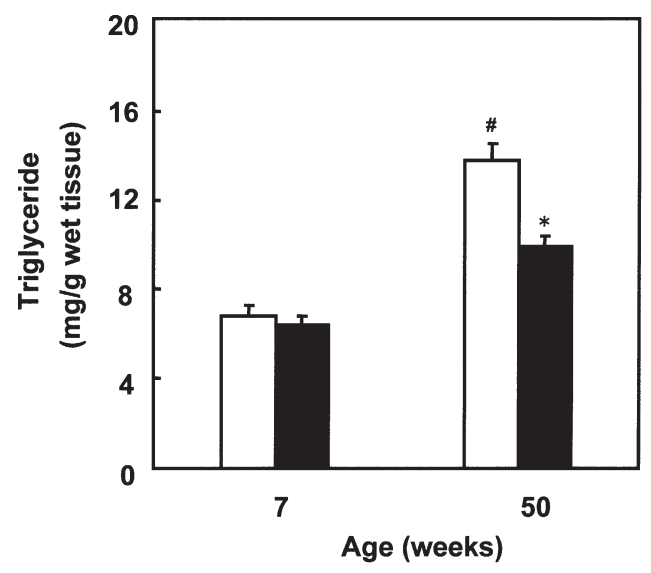

Figure 3. Change in triglyceride content in the liver tissues of normal or regucalcin transgenic (TG) rats. Rats were sacrificed by bleeding at 7 or 50 weeks of age. Each value is the mean \pm SEM of six rats. ${ }^{*} p<0.01$ compared with the control value from normal rats. ${ }^{*} p<0.01$ compared with the value obtained at 7 weeks of normal rats. White bars, normal rats; black bars, TG rats. 


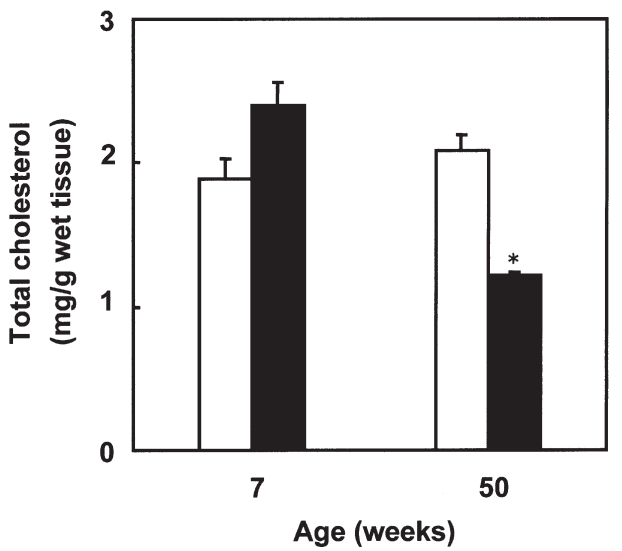

Figure 4. Change in total cholesterol content in the liver tissues of normal or regucalcin transgenic (TG) rats. Rats were sacrificed by bleeding at 7 or 50 weeks of age. Each value is the mean \pm SEM of six rats. ${ }^{*} p<0.01$ compared with the control value from normal rats. White bars, normal rats; black bars, TG rats.

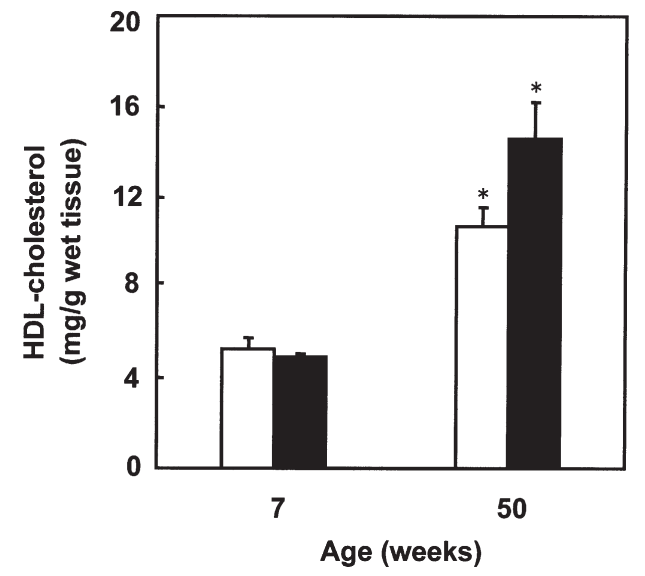

Figure 5. Change in HDL-cholesterol content in the liver tissues of normal or regucalcin transgenic (TG) rats. Rats were sacrificed by bleeding at 7 or 50 weeks of age. Each value is the mean \pm SEM of six rats. "p $<0.01$ compared with the value obtained at 7 weeks of normal rats. White bars, normal rats; black bars, TG rats.

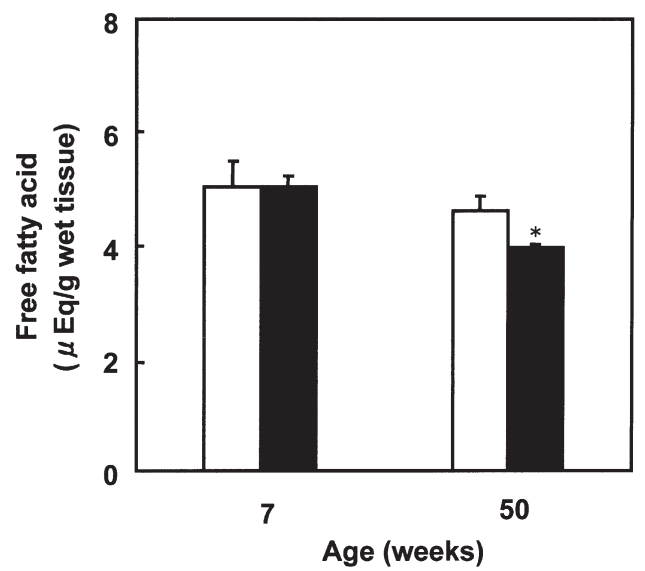

Figure 6. Change in free fatty acid content in the liver tissues of normal or regucalcin transgenic (TG) rats. Rats were sacrificed by bleeding at 7 or 50 weeks of age. Each value is the mean \pm SEM of six rats. ${ }^{*} \mathrm{p}<0.01$ compared with the control value from normal rats. White bars, normal rats; black bars, TG rats.

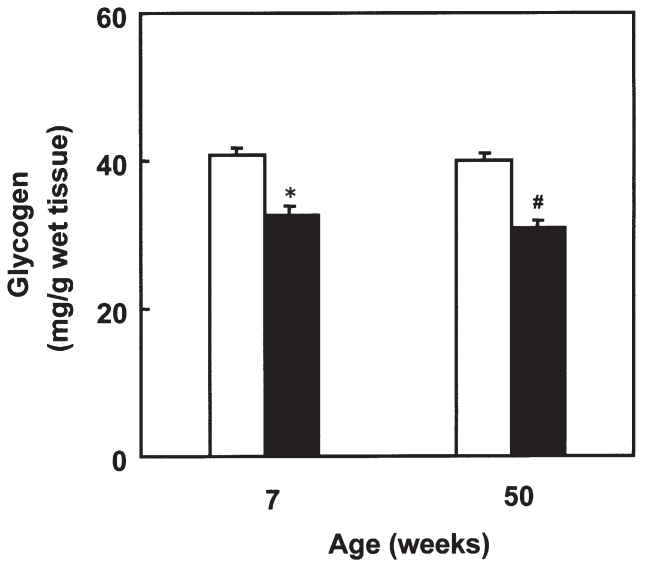

Figure 7. Change in glycogen content in the liver tissues of normal or regucalcin transgenic (TG) rats. Rats were sacrificed by bleeding at 7 or 50 weeks of age. Each value is the mean \pm SEM of six rats. ${ }^{*}, \mathrm{p}<<0.01$ compared with the control value from normal rats. White bars, normal rats; black bars, TG rats.
A mRNA

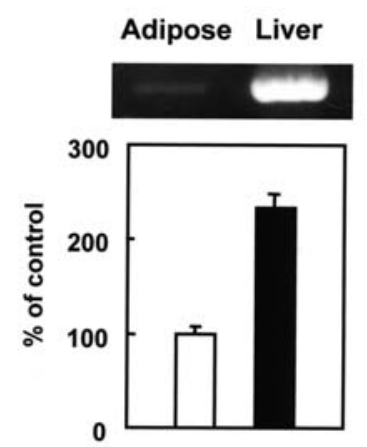

B Protein

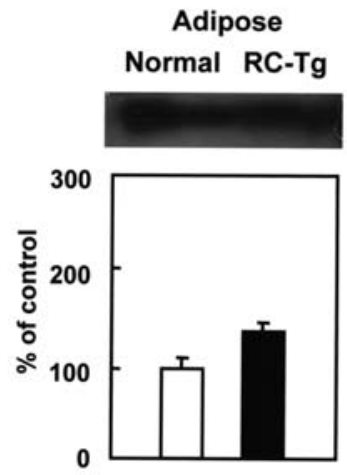

Figure 8. Expression of regucalcin mRNA and its protein in the adipose tissues of normal rats. Rats were sacrificed by bleeding at 50 weeks of age. The figure shows one of three experiments with separate rats.

HDL-cholesterol content was not significantly changed in 7- or 50-week-old regucalcin TG rats (Fig. 6). Liver HDLcholesterol content was significantly raised in 50-week-old normal or regucalcin TG rats as compared with that in 7-weekold normal or regucalcin TG rats, respectively.

Glycogen content in the liver tissues was significantly decreased in 7- or 50-week-old regucalcin TG rats as compared with that in 7- or 50-week-old normal rats, respectively (Fig. 7).

Change in leptin or adiponectin mRNA expression in the adipose or liver tissues of regucalcin TG rats. The expression of regucalcin mRNA and its protein in the adipose tissues of 50-week-old normal rats is shown in Fig. 8. Regucalcin mRNA and its protein levels were found in 50-week-old normal rats using RT-PCR and Western blot analysis (Fig. 8). Regucalcin protein levels were slightly increased in the adipose tissues of regucalcin TG rats as compared with that of 50-week-old normal rats (Fig. 8B).

The change in leptin mRNA levels in the adipose or liver tissues of regucalcin TG rats is shown in Fig. 9. The adipose or liver leptin mRNA levels were significantly decreased in 

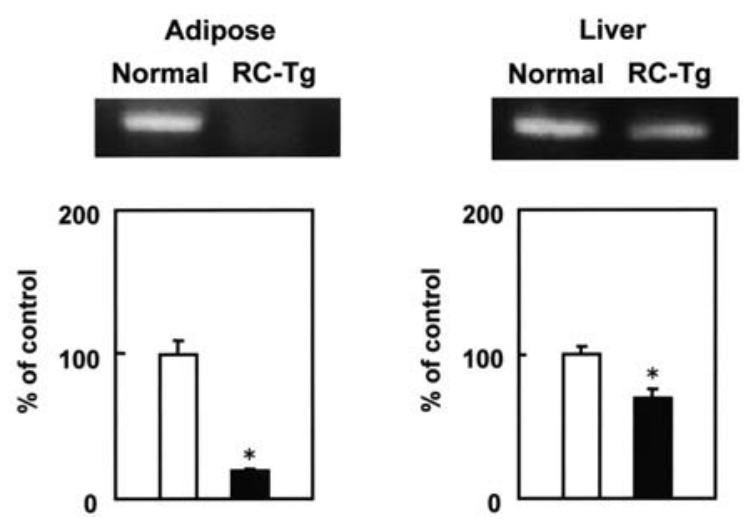

Figure 9. Change in leptin mRNA levels in the adipose and liver tissues of normal or regucalcin transgenic (TG) rats. Rats were sacrificed by bleeding at 50 weeks of age. The figure shows one of four experiments with separate rats. The densitometric data for mRNA levels are indicated as $\%$ of the value obtained from normal rats. ${ }^{*} \mathrm{p}<0.01$ compared with the value of normal rats.
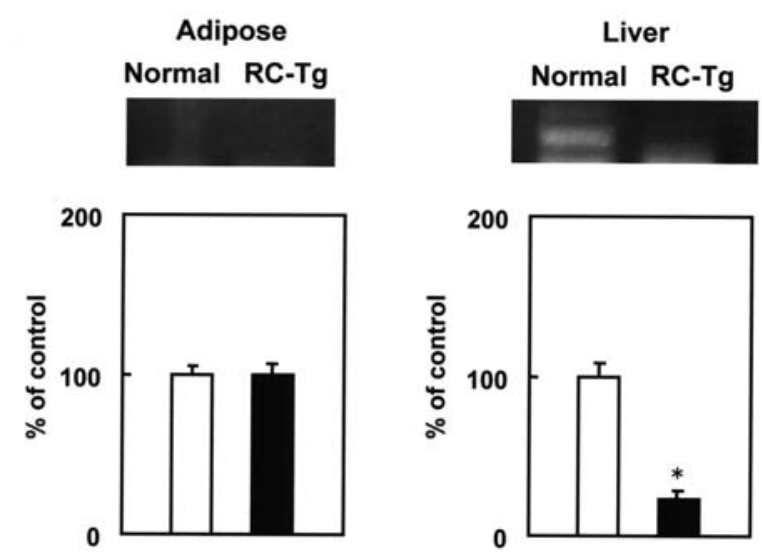

Figure 10. Change in adiponectin mRNA levels in the adipose and liver tissues of regucalcin transgenic (TG) rats. Rats were sacrificed by bleeding at 50 weeks of age. The figure shows one of four experiments with separate rats. The densitometric data for mRNA levels are indicated as $\%$ of the value obtained from normal rats. ${ }^{*} \mathrm{p}<0.01$ compared with the value of normal rats.

50-week-old regucalcin TG rats as compared with that of 50-week-old normal rats.

Adiponectin mRNA levels in the adipose tissues were not significantly changed in 50-week-old regcualcin TG rats (Fig. 10). This expression was significantly decreased in the liver tissues of 50-week-old regucalcin TG rats.

\section{Discussion}

Hyperlipidemia has been shown to occur in regucalcin TG rats with increasing age (30). This was confirmed in the present study. Triglyceride content in the adipose tissues was found to increase significantly in regucalcin TG rats, while triglyceride, total cholesterol, and free fatty acid contents in the liver tissues were significantly decreased in regucalcin TG rats. Hyperglycemia induced in regucalcin TG rats may be involved in the change in lipid components in the adipose and liver tissues of regucalcin TG rats.
Serum triglyceride concentration was significantly increased in regucalcin TG rats at 7 weeks of age as compared with that of normal rats. Triglyceride contents in the adipose and liver tissues were not significantly changed in regucalcin TG rats at 7 weeks of age. Meanwhile, glycogen content in the liver tissues was significantly decreased in regucalcin TG rats at 7 weeks of age, suggesting that hepatic glycogen synthesis is suppressed in regucalcin TG rats. The expression of regucalcin in liver tissue was significantly enhanced in regucalcin TG rats (29). Regucalcin has been shown to have a suppressive effect on glycogen phosphorylase $a$ activity in the glycogen particulate prepared from rat liver (38). It is speculated that regucalcin suppresses glycogen synthesis in the liver, and that the protein stimulates glycogenolysis in regucalcin TG rats. As the result, lipid synthesis may be stimulated in the liver tissues of regucalcin TG rats. Overexpression of regucalcin enhanced glucose utilization and lipid production in cloned rat hepatoma H4-II-E cells in vitro (39). In addition, regucalcin has been suggested to be involved in insulin resistance in $\mathrm{H} 4$ II-E cells $(39,40)$.

Regucalcin was expressed in the adipose tissues of normal rats. However, the enhancement of regucalcin expression in the adipose tissues was not seen in TG rats. Whether regucalcin in the adipose tissues stimulates the release of lipid components into the serum in regucalcin TG rats is unknown. Tryglyceride content in the adipose tissues was significantly increased in regucalcin TG rats. Presumably, an increase in serum lipid components may partly result from the release of lipids increased in the adipose tissues.

Leptin and adiponectin are adipokines that are involved in lipid metabolism $(41,42)$. The expression of leptin mRNA in the adipose and liver tissues was found to decrease significantly in regucalcin TG rats. Adiponectin mRNA expression was significantly suppressed in the livers of regucalcin TG rats. Leptin or adiponectin regulates hyperlipidemia $(43,44)$. These decreases may be partly involved in the induced hyperlipidemia in regucalcin TG rats.

Regucalcin TG rats exhibited an increase in serum lipid components, a decrease in hepatic lipid and glycogen contents, and a suppression of leptin mRNA expression in their adipose and liver tissues. Thus, the disorder of lipid metabolism was induced in regucalcin TG rats, indicating the usefulness of this animal model in studying this disorder.

In conclusion, it has been shown that a decrease in lipid and glycogen contents in liver tissue is induced in regucalcin TG rats, and that the gene expression of leptin and adiponectin is suppressed in TG rats.

\section{References}

1. Yamaguchi $\mathrm{M}$ and Yamamoto T: Purification of calcium binding substance from soluble fraction of normal rat liver. Chem Pharm Bull 26: 1915-1918, 1978.

2. Yamaguchi $M$ and Sugii K: Properties of calcium-binding protein isolated from the soluble fraction of normal rat liver. Chem Pharm Bull 29: 567-570, 1981.

3. Yamaguchi M: Physicochemical properties of calcium-binding protein isolated from rat liver cytosol: $\mathrm{Ca}^{2+}$-induced conformational changes. Chem Pharm Bull 36: 286-290, 1988.

4. Yamaguchi M and Mori S: Effects of $\mathrm{Ca}^{2+}$ and $\mathrm{Zn}^{2+}$ on 5'nucleotidase activity in rat liver plasma membranes: Hepatic calcium-binding protein (regucalcin) reverses the $\mathrm{Ca}^{2+}$ effect. Chem Pharm Bull 36: 321-325, 1988. 
5. Shimokawa $\mathrm{N}$ and Yamaguchi $\mathrm{M}$ : Molecular cloning and sequencing of the cDNA coding for a calcium-binding protein regucalcin from rat liver. FEBS Lett 327: 251-255, 1993.

6. Yamaguchi M: A novel $\mathrm{Ca}^{2+}$-binding protein regucalcin and calcium function. In: Calcium Inhibition. Kohama K (ed). Japan Sci Soc Press, Tokyo and CRC Press, Boca Raton, pp19-41, 1992.

7. Yamaguchi M: Role of regucalcin in calcium signaling. Life Sci 66: 1769-1780, 2000.

8. Yamaguchi M: Role of regucalcin in nuclear regulation of regenerating liver. Biochem Biophys Res Commun 276: 1-6, 2000.

9. Yamaguchi M: Role of regucalcin in maintaining cell homeostasis and function. Int J Mol Med 15: 372-389, 2005.

10. Misawa $\mathrm{H}$ and Yamaguchi M: The gene of $\mathrm{Ca}^{2+}$-binding protein regucalcin is highly conserved in vertebrate species. Int $\mathbf{J}$ Mol Med 6: 191-196, 2000.

11. Shimokawa N, Matsuda Y and Yamaguchi M: Genomic cloning and chromosomal assignment of rat regucalcin gene. Mol Cell Biochem 151: 157-163, 1995.

12. Thiselton DL, McDowall J, Brandau O, Ramsere J, d'Esposito F, Bhattacharya SS, Ross MT, Hardcastle AJ and Meaindl A: An integrated, functionally annotated gene map of the DXS80269FLK1 interval on human Xp11.3-Xp11.23: Potential hotspot for neurogenetic disorders. Genomics 79: 560-572, 2002.

13. Yamaguchi $\mathrm{M}$ and Isogai M: Tissue concentration of calciumbinding protein regucalcin in rats by enzyme-linked immunoadsorbent assay. Mol Cell Biochem 122: 65-68, 1993.

14. Shimokawa N and Yamaguchi M: Calcium administration stimulates the expression of calcium-binding protein regucalcin mRNA in rat liver. FEBS Lett 305: 151-154, 1992.

15. Murata T and Yamaguchi M: $\mathrm{Ca}^{2+}$ administration stimulates the binding of AP-1 factor to the 5'-flanking region of the rat gene for the $\mathrm{Ca}^{2+}$-binding protein regucalcin. Biochem $\mathrm{J}$ 329: 157-183, 1998.

16. Murata $\mathrm{T}$ and Yamaguchi M: Promoter characterization of the rat gene for $\mathrm{Ca}^{2+}$-binding protein regucalcin. Transcriptional regulation by signaling factors. J Biol Chem 274: 1277-1285, 1999.

17. Misawa $\mathrm{T}$ and Yamaguchi M: Identification of transcriptional factor in the promoter region of rat regucalcin gene: binding of nuclear factor I-A1 to TTGGC motif. J Cell Biochem 84: 795-802, 2002.

18. Sawada N and Yamaguchi M: Overexpression of RGPR-p117 enhances regucalcin gene promoter activity in cloned normal rat kidney proximal tubular epithelial cells: Involvement of TTGGC motif. J Cell Biochem 99: 589-597, 2006.

19. Shimokawa N and Yamaguchi M: Expression of hepatic calcium-binding protein regucalcin mRNA is mediated through $\mathrm{Ca}^{2+} /$ calmodulin in rat liver. FEBS Lett 316: 79-84, 1993

20. Yamaguchi $M$ and Nakajima M: Involvement of intracellular signaling factors in the serum-enhanced $\mathrm{Ca}^{2+}$-binding protein regucalcin mRNA expression in the cloned rat hepatoma cells (H4-II-E). J Cell Biochem 74: 81-89, 1999.

21. Tsurusaki Y and Yamaguchi M: Suppressive role of endogenous regucalcin in the enhancement of deoxyribonucleic acid synthesis activity in the nucleus of regenerating rat liver. J Cell Biochem 85: 516-522, 2002.

22. Tsurusaki Y and Yamaguchi M: Role of endogenous regucalcin in nuclear regulation of regenerating rat liver: Suppression of the enhanced ribonucleic acid synthesis activity. J Cell Biochem 87: 450-457, 2002.

23. Tsurusaki Y and Yamaguchi M: Overexpression of regucalcin modulates tumor-related gene expression in cloned rat hepatoma H4-II-E cells. J Cell Biochem 90: 619-626, 2003.

24. Misawa H, Inagaki S and Yamaguchi M: Suppression of cell proliferation and deoxyribonucleic acid synthesis in cloned rat hepatoma H4-II-E cells overexpressing regucalcin. J Cell Biochem 84: 143-149, 2002.
25. Yamaguchi $M$ and Sakurai T: Inhibitory effect of calcium-binding protein regucalcin on $\mathrm{Ca}^{2+}$-activated DNA fragmentation in rat liver nuclei. FEBS Lett 279: 281-284, 1991.

26. Izumi $\mathrm{T}$ and Yamaguchi M: Overexpression of regucalcin suppresses cell death in cloned rat hepatoma H4-II-E cells induced by tumor necrosis factor- $\alpha$ or thapsigargin. J Cell Biochem 92: 296-306, 2004.

27. Nakagawa $\mathrm{T}$ and Yamaguchi M: Overexpression of regucalcin suppresses apoptotic cell death in cloned normal rat kidney proximal tubular epithelial NRK52E cells: Change in apoptosisrelated gene expression. J Cell Biochem 96: 1274-1285, 2005.

28. Yamaguchi M, Morooka Y, Misawa H, Tsurusaki Y and Nakajima R: Role of endogenous regucalcin in transgenic rats: suppression of kidney cortex cytosolic protein phosphatase activity and enhancement of heart muscle microsomal $\mathrm{Ca}^{2+}$ ATPase activity. J Cell Biochem 86: 520-529, 2002.

29. Yamaguchi M, Misawa H, Uchiyama S, Morooka Y and Tsurusaki Y: Role of endogenous regucalcin in bone metabolism: Bone loss is induced in regucalcin transgenic rats. Int J Mol Med 10: 377-383, 2002.

30. Yamaguchi M, Isogai A, Uchiyama S and Sawada N: Hyperlipidemia is induced in regucalcin transgenic rats with increasing age. Int J Mol Med 14: 647-651, 2004.

31. Good CA, Kramer H and Somogyi M: The determination of glycogen. J Biol Chem 100: 485-491, 1933.

32. Laemmli UK: Cleavage of structural proteins during the assembly of the hand of bacteriophage T4. Nature 22: 680-685, 1970 .

33. Wessendolf JHM, Ganfinkel S, Zhan X, Brown S and Maciag T: Identification of a nuclear localization sequence within the structure of the human interleukin-1 $\alpha$ precursor. J Biol Chem 268: 22100-22104, 1993.

34. Lowry OH, Rosebrogh NH, Farr AL and Randall RF: Protein measurement with the Folin phenol reagent. J Biol Chem 193: 265-273, 1951.

35. Chomczyshi P and Sacchi N: Single-step method of RNA isolation by acid guanidium thiocyanate-phenol-chloroform extraction. Anal Biochem 162: 156-159, 1987.

36. Murakami T and Shima K: Cloning of rat obese cDNA and its expression in obese rats. Biochem Biophys Res Commun 209: 994-952, 1995.

37. Puerta M, Abelenda M, Roche M and Trayhurn P: Effect of acute cold exposure on the expression of the adiponectin, resistin and leptin genes in rat white and brown adipose tissues. Horm Metab Res 34: 624-634, 2002.

38. Yamaguchi M and Shibano H: Effect of calcium-binding protein on the activation of phosphorylase $a$ in rat hepatic particulate glycogen by $\mathrm{Ca}^{2+}$. Chem Pharm Bull 35: 2581-2584, 1987.

39. Nakashima $C$ and Yamaguchi M: Overexpression of regucalcin enhances glucose utilization and lipid production in cloned rat hepatoma H4-II-E cells: Involvement of insulin resistance. J Cell Biochem 99: 1582-1592, 2006.

40. Soloman SS, Buss N, Shull J, Monnier S, Majumdar G, Wu J and Gerling IC: Proteome of H4-II-E (liver) cells exposed to insulin and tumor necrosis factor- $\alpha$ : Analysis of proteins involved in insulin resistance. J Lab Clin Med 145: 275-283, 2005.

41. Ronti T, Lupattelli G and Mannarino E: The endocrine function of adipose tissue: an update. Clin Endocrinol 64: 355-365, 2006.

42. Dyck DJ, Heigenhauser GJ and Bruce CR: The role of adipokines as regulators of skeletal muscle fatty acid metabolism and insulin sensitivity. Acta Physiol 186: 5-16, 2006.

43. Guerre-Millo M: Adipose tissue hormones. J Endocrinol Invest 25: 855-861, 2002.

44. Havel PJ: Update on adipocyte hormones: regulation of energy balance and carbohydrate/lipid metabolism. Diabetes 53: 5143-5151, 2004. 